\title{
État des lieux des technologies innovantes dans la e-santé
}

Dominique Leleu

$>$ Cette présentation repose sur mon expérience en tant que dirigeant d' «Interaction Healthcare » qui est un acteur dans le domaine de la e-santé, et en tant que membre du Think Tank «Renaissance Numérique », qui réfléchit à la place du numérique au service du citoyen. Un groupe de travail est dédié à la santé. Elle doit aussi à la Commission Service de «Cap Digital », qui s'intéresse à la rencontre des acteurs des mondes académique, industriel et des start-ups. Tous ces acteurs cherchent à imaginer le numérique et à comprendre les innovations qui nous entourent, dans l'intérêt des patients et des professionnel de la santé, car la e-santé doit devenir plus concrète, par exemple dans l'utilisation de la simulation numérique pour la formation en santé.

La e-santé revêt une partie technologique et porte, par exemple, sur la robotique, les applications de m-santé, la télémédecine et les systèmes d'information. II s'agit d'une grande famille de solutions technologiques au service des différents niveaux d'intervention en santé.

\section{Le marché de la e-santé et ses tendances}

Le potentiel du marché de la e-santé en France est estimé entre 2,2 milliards d'euros et 3 milliards d'euros actuellement, et pourrait atteindre 4 milliards d'euros en 2020. Des actions ont également été menées par les ARS. 80 millions d'euros ont été investis pour soutenir le secteur de la e-santé dans le cadre du programme «Territoires de soins numérique ». 30 \% des acteurs du marché sont des start-ups, qui réalisent moins d'un million d'euros de chiffre d'affaires. Ce secteur est en pleine expansion.

Les grandes tendances de la e-santé portent sur le collaboratif, les plateformes d'accompagnement, les objets connectés et wearables, les nanotechnologies, la gamification, la réalité augmentée et virtuelle, l'intelligence artificielle, l'impression 3D et la médecine personnalisée et prédictive. C'est bien dans cette vision systémique que nous devons nous inscrire, afin de comprendre les enjeux de la e-santé.

On parle par ailleurs souvent de la médecine des $4 \mathrm{P}$ (Prédictive, Préventive, Personnalisée, Participative), mais celle des 5 P (basée sur les Preuves) est maintenant de plus en plus évoquée. L'ensemble des acteurs de la e-santé s'inscrit maintenant dans une médecine des preuves.

L'écosystème de la e-santé est nouveau aujourd'hui. Parmi les acteurs qui fournissent des solutions, on trouve des acteurs du numérique présents depuis longtemps, des mutuelles, des laboratoires, des acteurs du numérique comme Cisco, ainsi que les GAFA (Google, Amazon, Facebook et Apple) qui essaient de préempter le domaine de la santé, avec plus ou moins de succès.

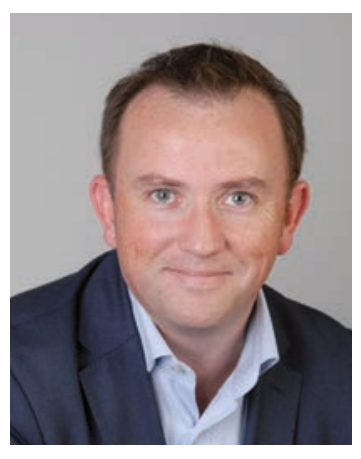

Président Interaction

Healthcare, Interaction Healthcare, 134, rue Danton, 92300 Levallois-Perret, France. jerome.leleu@interactionhealthcare.com

Ces acteurs ne travaillent pas en silo mais de manière collaborative. Des collaborations ont ainsi été envisagées avec 23 andMe qui permet d'obtenir des informations sur le génome et les prédispositions génétiques.

Sanofi et Verily (anciennement Google Life Sciences), ont créé une joint-venture sur la prise en charge du diabète. Dans la robotique, des acteurs comme Google travaillent avec Ethicon pour proposer des plateformes d'intelligence artificielle permettant d'améliorer la prise en charge du chirurgien et du patient. Ces technologies permettent de faire gagner du temps et de ne rien oublier. Des questions se posent toutefois, en termes d'éthique, de collecte de données et d'information. Un cadre précis doit donc être défini.

Le téléphone mobile constitue un élément intéressant pour le patient. Nous avons constaté une forte évolution des applications ces dernières années. Les fonctionnalités du mobile sont utilisées pour accompagner le patient et permettre un meilleur échange avec les professionnels de santé. Ces technologies ne vont pas remplacer le médecin, mais renforcer la relation avec le patient en lui accordant plus de temps.

On parle aussi des médias sociaux. Certains réseaux, comme Facebook, se développent. On compte également des réseaux sociaux thématiques comme Carneti, PatientsLikeMe et My Hospi Friends. Ces réseaux permettent de partager des informations et des expériences dans le milieu hospitalier. Ils favorisent les échanges entre patients et encouragent la circulation de l'information et des pratiques.

Une initiative a été lancée il y a quelques années dans la Silicon Valley, intitulée CrowdMed. Dans le cadre d'un documentaire diffusé sur Arte, une patiente témoignait 
de l'intérêt de cette initiative. Lorsqu'un patient se trouve dans une situation d'errance de diagnostic, il peut publier son cas directement, de manière anonyme, et le mettre à disposition de l'ensemble de la communauté médicale. L'aspect éthique doit être pris en compte dans ce type de démarche.

\section{Les nouveaux outils}

S'agissant des essais cliniques, une étude présentée à Boston a démontré que les propositions et récoltes d'information par Facebook étaient plus pertinentes que d'autres, s'agissant du nombre de volontaires inclus.

Par ailleurs, les serious games peuvent permettre à des enfants de mieux comprendre leur maladie et à leurs parents de mieux les accompagner. Ce type de jeux peut également donner des outils aux enseignants, dans le cadre d'une approche pédagogique.

En cas d'errance diagnostique, un accompagnement est nécessaire par la simulation numérique. Il est ainsi possible, par exemple, de prendre en charge un patient atteint d'accidents vasculaires cérébraux dans toutes les étapes. Le raisonnement clinique peut permettre de comprendre comment le patient doit être accompagné de son domicile à l'hôpital et en centre de rééducation.

IBM s'implique par exemple fortement dans l'intelligence artificielle. Il est ainsi possible de disposer d'un recueil d'informations consultables dans des bases extrêmement importantes et internationales, qui ne pourrait jamais être constitué par un médecin. Aujourd'hui, ce type d'outil doit encore s'améliorer, mais peut aider le médecin à prendre une décision plus rapidement et à passer plus de temps avec le patient.

Les Natou sont Netflix, Airbnb, Telsa Motors et Uber. Uber permet de se déplacer dans de nombreuses villes du monde, mais propose parfois d'autres solutions. En Inde, Uber $X$ permet de livrer des médicaments à domicile.

La e-santé ne doit pas s'enfermer dans une démarche trop technologique et réglementaire, mais favoriser l'échange entre les patients et les professionnels et apporter des solutions utiles. Les acteurs numériques, souvent américains, ont trouvé des solutions. De nombreux acteurs du monde hospitalier et de la recherche souhaitent par ailleurs pouvoir proposer ce type de solutions.

Pour conclure, un élément me semble essentiel. Le cœur de la e-santé, ce sont les lieux de rencontre entre médecins, développeurs, ingénieurs, pharmaciens et patients qui vont imaginer la santé de demain. L'innovation de demain en santé se conçoit avant tout avec les patients et les professionnels de santé. $\diamond$

State of play of e-health technologies

\section{LIENS D'INTÉRÊT}

L'auteur déclare n'avoir aucun lien d'intérêt concernant les données publiées dans cet article.

\section{État des lieux des expérimentations innovantes en télémédecine en France}

\section{Bilan 2018 : une évolution progressive vers la maturité du déploiement technologique}

Mon expérience est celle d'un praticien interniste, urgentiste, qui travaille dans la télémédecine (TLM) depuis 2004. J'ai acquis un regard à travers ce qui se passe dans différentes régions, chaque région étant organisée en silo. Les silos entre spécialités et la médecine de ville se sont reproduits dans les systèmes d'information et de communication. Comment faire pour dépasser cela?

La première étape est de disposer de l'outil de visioconférence. Développer des réunions de concertation pluridisciplinaires entre centres de

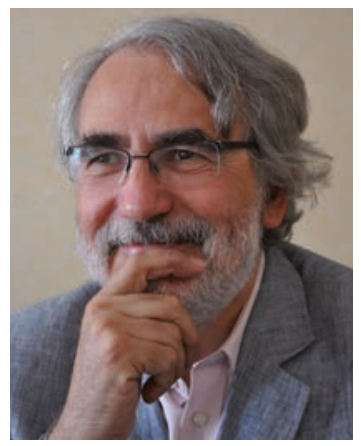

Consultant espinoza.telemedecine@ gmail.com www.sf-telemed.org

référence, centres de compétences et médecins traitants est un objectif prioritaire. Ces réunions favoriseront les transferts de savoir et permettront de conforter une rela- 